\title{
Inflamabilidade de espécies arbóreas para uso em cortinas de segurança na prevenção de incêndios florestais
}

\author{
Bruna Kovalsyki ${ }^{1}$, Igor Kiyoshi Takashina ${ }^{1 *}$, Andressa Tres ${ }^{1}$, Alexandre França Tetto $^{1}$, Antonio Carlos Batista ${ }^{1}$
}

Universidade Federal do Paraná, Av. Pref. Lothário Meissner, 632, CEP 80210-170, Curitiba, PR, Brasil

"Autor correspondente:

igor.takashina@gmail.com

Termos para indexação:

Aceiro verde

Proteção florestal

Silvicultura preventiva

Index terms:

Fuelbreaks

Forest protection

Preventive Forestry

Histórico do artigo:

Recebido em 27/07/2015

Aprovado em 29/08/2016

Publicado em 30/12/2016

doi: 10.4336/2016.pfb.36.88.991
Resumo - Dentre as medidas silviculturais de prevenção contra incêndios florestais destacam-se as cortinas de segurança. Estas estruturas consistem em plantios com espécies de menor inflamabilidade em relação ao cultivo principal, que tem como objetivo reduzir e/ou prevenir a propagação do fogo. O objetivo deste estudo foi avaliar a inflamabilidade de Psidium cattleianum Sabine., Ligustrum lucidum W. T. Aiton., Schinus terebinthifolius Raddi. e Bougainvillea glabra Choisy., para potencial uso em cortinas de segurança. Nesta pesquisa utilizou-se Pinus taeda L. como controle. As queimas foram realizadas em epirradiador, com temperatura de $250{ }^{\circ} \mathrm{C}$ a $350{ }^{\circ} \mathrm{C}$, para as quais se utilizou $1 \mathrm{~g}$ de material fino $(<0,7 \mathrm{~cm}$ de diâmetro) recém-coletado. Para cada espécie, realizou-se 50 repetições, sendo analisados: frequência de ignição, tempo para ignição, duração da combustão, índice de combustão, além da determinação do valor de inflamabilidade. P. taeda apresentou uma intensidade de combustão muito alta e foi classificado como espécie inflamável. As demais espécies foram consideradas fracamente inflamáveis, sendo que $S$. terebinthifolius e B. glabra indicaram uma intensidade de combustão baixa, L. lucidum média e $P$. cattleianum, alta. Neste contexto, conclui-se que as espécies avaliadas apresentam potencial para uso em cortina de segurança na prevenção de incêndios florestais.

\section{Flammability of tree species to be used in fuelbreaks at forest fires prevention}

\begin{abstract}
Among several silvicultural measures of forest fires prevention, fuelbreaks stands out. These structures are used to reduce and/or prevent fire spread. They consist of plantations with lower flammability species than the main species. The aim of this study was to evaluate the flammability of Psidium cattleianum Sabine., Ligustrum lucidum W. T. Aiton., Schinus terebinthifolius Raddi. and Bougainvillea glabra Choisy, for potential use in fuelbreaks. In this research Pinus taeda L. was used as control. Samples consisted of $1 \mathrm{~g}$ of fine material $(<0.7 \mathrm{~cm}$ of diameter) newly collected. Samples burning were performed in epiradiator, under temperature between $250^{\circ} \mathrm{C}$ and $350^{\circ} \mathrm{C}$. It was carried out 50 replications for each species. It was analyzed ignition frequency, time to ignition, combustion duration, combustion index, and it was also determined flammability value. $P$. taeda presents a very high combustion intensity and was classified as a flammable species. The other species were considered poorly flammable. S. terebinthifolius and B. glabra indicated low combustion intensity, L. lucidum medium and P. cattleianum high combustion intensity. In this context, it was concluded that these species have potential to be used in fuelbreaks to prevent forest fires.
\end{abstract}




\section{Introdução}

As ocorrências dos incêndios florestais, principalmente os de grande magnitude, causam prejuízos econômicos, ecológicos e paisagísticos, além de ameaçarem a vida humana. Segundo Soares (2000), para diminuir a ocorrência dos incêndios o ideal é adotar uma silvicultura preventiva, ou seja, estabelecer técnicas de proteção contra o fogo desde a implantação dos povoamentos, uma vez que estes são potencialmente mais suscetíveis aos incêndios.

Segundo Haltenhoff (2006), a silvicultura preventiva é o conjunto de práticas adotadas para modificar a estrutura do combustível disponível e, assim, atender aos objetivos de proteção contra incêndios florestais, visando melhor produção e qualidade do ambiente.

Uma das medidas de prevenção de incêndios florestais propostas pela silvicultura preventiva é a implantação de cortinas de segurança. As cortinas de segurança são plantios em faixas compostas por espécies de menor inflamabilidade do que o cultivo principal, com o objetivo de reduzir e/ou prevenir a propagação do fogo, facilitando, desta maneira, o controle e o combate dos incêndios (Batista \& Biondi, 2009). Conforme Hernando (2009), a inflamabilidade está associada à facilidade com que o material combustível entra em ignição, a propriedade do combustível em continuar queimando, a velocidade de queima e a quantidade de combustível consumido.

Ribeiro et al. (2007), em testes de espécies para compor cortinas de segurança sob uma linha de transmissão de energia elétrica, apontaram como espécies potenciais Hibiscus sp. (hibisco) e Mimosa caesalpiniifolia (sansãodo-campo). De acordo com os autores, considera-se importante na seleção das espécies para compor uma cortina de segurança, características como: potencial produtivo, tolerância à seca e perenidade, além de alto teor de umidade, copa densa, crescimento rápido e folhas coriáceas. Tendo essas características em vista, foram selecionadas quatro espécies arbóreas para a realização deste estudo, o qual teve como objetivo avaliar a inflamabilidade e indicar a potencialidade das mesmas para uso na implantação de cortinas de segurança.

\section{Material e métodos}

Todos os experimentos e análises necessários para desenvolvimento da pesquisa foram realizados no Laboratório de Proteção Florestal do Departamento de
Ciências Florestais da Universidade Federal do Paraná, de acordo com a metodologia apresentada por Valette (1992) e Petriccione (2006).

As espécies arbóreas avaliadas foram: Psidium cattleianum Sabine. (araçá), Ligustrum lucidum W. T. Aiton (alfeneiro), Schinus terebinthifolius Raddi. (aroeira) e Bougainvillea glabra Choisy. (buganvile). Pinus taeda L. foi utilizado como controle, por ser considerado inflamável e por ser muito cultivado na região sul do Brasil.

Foram coletados folhas e ramos com diâmetro inferior a $0,7 \mathrm{~cm}$, considerados como combustível fino das copas das espécies previamente selecionadas (Soares \& Batista, 2007). A determinação do teor de umidade foi feita utilizando-se a equação 1 (Batista, 1990), sendo a massa seca obtida por meio de subamostras de $200 \mathrm{~g}$, secas em estufa a $75^{\circ} \mathrm{C}$, por $48 \mathrm{~h}$.

$$
U \%=\frac{M U-M S}{M S} \cdot 100
$$

Sendo: $U \%=$ teor de umidade do material combustível (\%); $M U=$ massa úmida do combustível no momento da coleta (g); e $M S=$ massa seca do material combustível (g).

O material combustível foi utilizado em um prazo máximo de $2 \mathrm{~h}$ após a coleta, visto que após este período as condições das amostras não estariam em seu estado natural. As queimas experimentais foram realizadas no epirradiador, com uma faixa de temperatura controlada de $250{ }^{\circ} \mathrm{C}$ a $350{ }^{\circ} \mathrm{C}$. Este equipamento necessita de condições ideais para um bom funcionamento. Para isso, o mesmo foi instalado em uma capela, local livre de corrente de ar, para garantir a homogeneidade das condições meteorológicas durante o experimento. Também foi necessária a existência de uma chama piloto, localizada no centro do disco e a $4 \mathrm{~cm}$ acima do mesmo (Figura 1).

Para cada espécie avaliada, efetuou-se 50 repetições de queima. Cada queima era constituída de $1 \pm 0,1 \mathrm{~g}$ de material combustível verde, determinado com auxílio de uma balança de precisão. Todas as etapas foram realizadas sem o contato direto com o material, para evitar interferências em suas propriedades.

Foram analisadas as seguintes características da combustão, conforme proposto por Petriccione (2006):

i. tempo para ignição (TI): tempo que o material leva para iniciar a combustão; 

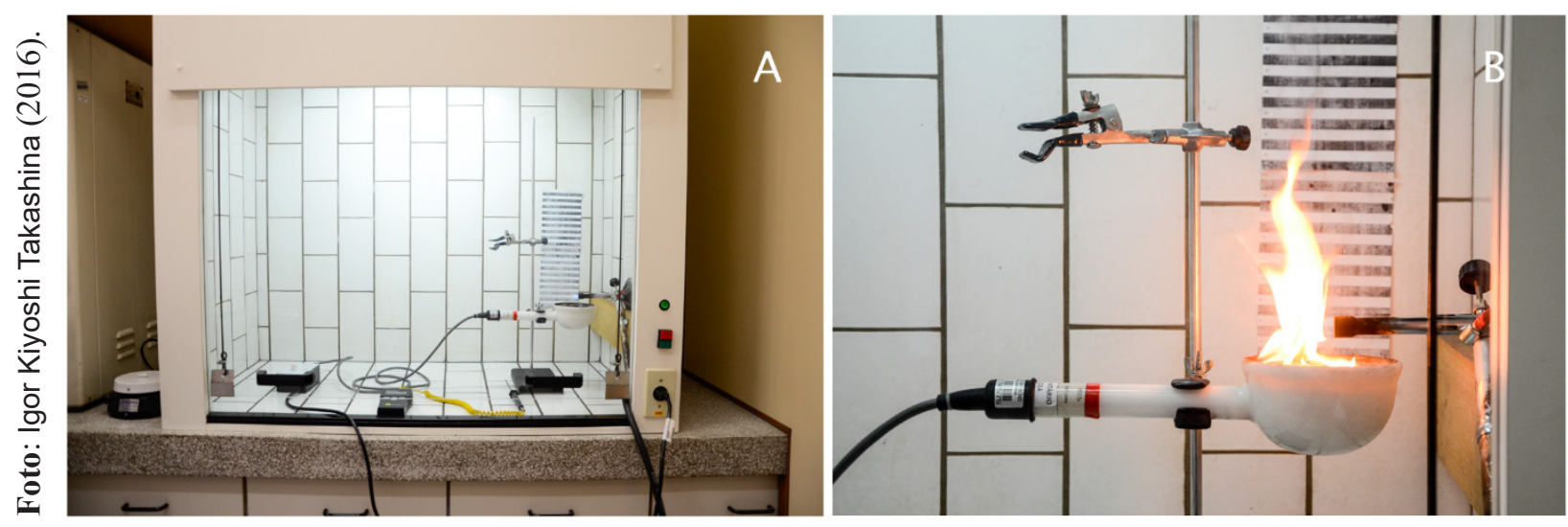

Figura 1. Demonstração do epirradiador na capela (A) e ensaio em epirradiador (B)

ii. frequência de ignição (FI): número de repetições em que ocorreu ignição, considerando um TI máximo de $60 \mathrm{~s}$, em queimas que ultrapassaram este tempo foram classificadas como "queimas negativas";

iii. duração da combustão (DC): tempo que a chama se mantém acesa;

iv. índice de combustão (IC): intensidade de combustão de cada queima, sendo determinada através da média das alturas de chama e classificada segundo os índices apresentados no Tabela 1.

v. valor de inflamabilidade (VI): atribuído de acordo com a FI e o TI (Tabela 2).

Tabela 1. Índices do valor de combustão.

\begin{tabular}{ccc}
\hline $\begin{array}{c}\text { Índice de } \\
\text { combustão (IC) }\end{array}$ & $\begin{array}{c}\text { Designação } \\
\text { do IC }\end{array}$ & $\begin{array}{c}\text { Comprimento da } \\
\text { chama }(\mathbf{c m})\end{array}$ \\
\hline IC1 & Muito baixa & $<1$ \\
IC2 & Baixa & 1 a 3 \\
IC3 & Média & 4 a 7 \\
IC4 & Alta & 8 a 12 \\
IC5 & Muito alta & $>12$ \\
\hline
\end{tabular}

Fonte: Petriccione (2006).

Tabela 2. Índices do valor de inflamabilidade.

\begin{tabular}{ccccccc}
\hline \multirow{2}{*}{ TI (s) } & \multicolumn{7}{c}{ FI } \\
\cline { 2 - 7 } & $<\mathbf{2 5}$ & $\mathbf{2 5 - 3 8}$ & $\mathbf{3 9 - 4 1}$ & $\mathbf{4 2 - 4 4}$ & $\mathbf{4 5 - 4 7}$ & $\mathbf{4 8 - 5 0}$ \\
\hline$>32,5$ & 0 & 0 & 0 & 1 & 1 & 2 \\
$27,6-32,5$ & 0 & 0 & 1 & 1 & 2 & 2 \\
$22,6-27,5$ & 0 & 0 & 1 & 2 & 2 & 2 \\
$17,6-22,5$ & 1 & 1 & 2 & 2 & 3 & 3 \\
$12,6-17,5$ & 1 & 1 & 2 & 3 & 3 & 4 \\
$<12,6$ & 1 & 2 & 3 & 3 & 4 & 5 \\
\hline
\end{tabular}

Nota: FI - frequência de ignição e TI - tempo para ignição. O valor de inflamabilidade (VI) será classificado em função do número correspondente, em que: VI $=0$ (fracamente inflamável); VI $=1$ (pouco inflamável); VI $=2$ (moderadamente inflamável); VI = 3 (inflamável); VI = 4 (altamente inflamável) ou VI = 5 (extremamente inflamável). Fonte: Valette (1992).
Procedeu-se a análise de variância (ANOVA) para cada espécie em relação a $P$. taeda. Foi utilizado o teste de Tukey ao nível de $5 \%$ de probabilidade para comparação entre médias, envolvendo todas as espécies. Para as queimas classificadas como negativas, considerou-se TI de $61 \mathrm{~s}$, duração de combustão de zero s e altura de chama de zero $\mathrm{cm}$.

Para a espécie ser considerada de uso potencial em cortinas de segurança, espera-se que os valores de frequência de ignição, duração da chama e de altura da chama sejam inferiores, quando comparados aos valores apresentados por P. taeda, e superiores para o valor de tempo de ignição.

\section{Resultados e discussão}

As espécies Psidium cattleianum, Ligustrum lucidum, Schinus terebinthifolius e Bougainvillea glabra apresentaram diferença significativa em relação a Pinus taeda. O teor de umidade, a frequência de ignição e os valores médios das variáveis de inflamabilidade das espécies analisadas estão apresentados na Tabela 3.

A espécie que apresentou os melhores resultados para as variáveis de inflamabilidade foi B. glabra. Entretanto, as quatro espécies estudadas apresentaram valores inferiores de duração de combustão, altura de chama e frequência de ignição e tempo de ignição superior aos valores apresentados por P. taeda.

Segundo Hernando (2009), as variáveis relacionadas com o fogo estão significativamente relacionadas com o teor de umidade dos combustíveis, principalmente o tempo de ignição e o comprimento inicial da chama. Além disso, Alessio et al. (2008) sugerem que o conteúdo de terpenos voláteis pode influenciar a inflamabilidade. 
Tabela 3. Valores médios das variáveis de inflamabilidade.

\begin{tabular}{lrrrrrrrr}
\hline \multirow{2}{*}{ Espécie } & \multicolumn{9}{c}{ Média } & \multirow{2}{*}{ DC } & HC & \multirow{2}{*}{ FI } \\
\cline { 2 - 6 } & \multicolumn{2}{c}{ TI } & & \\
\hline Pinus taeda & 18,4 & $\mathrm{~d}$ & 11,8 & $\mathrm{a}$ & 12,1 & $\mathrm{a}$ & 136,0 & 100 \\
Psidium cattleianum & 49,5 & $\mathrm{c}$ & 4,7 & $\mathrm{~b}$ & 7,9 & $\mathrm{~b}$ & 128,0 & 62 \\
Ligustrum lucidum & 54,2 & $\mathrm{~b}$ & 2,3 & $\mathrm{c}$ & 5,9 & $\mathrm{~b}$ & 158,7 & 36 \\
Schinus terebinthifolius & 58,4 & $\mathrm{ab}$ & 1,3 & $\mathrm{c}$ & 1,7 & $\mathrm{c}$ & 142,0 & 14 \\
Bongainvillea glabra & 59,6 & $\mathrm{a}$ & 0,7 & $\mathrm{c}$ & 1,2 & $\mathrm{c}$ & 210,6 & 10 \\
\hline
\end{tabular}

Nota: letras iguais indicam que, ao nível de $5 \%$, não há diferença significativa entre as médias. TI - tempo para ignição (s); DC - duração da combustão (s); HC - altura da chama (cm); U\% - teor de umidade (\%); FI - frequência de ignição (\%).

Neste sentido, observou-se correlação entre o teor de umidade (U\%) e os parâmetros: tempo médio para ignição (MTI) $(\mathrm{r}=0,7)$, tempo médio da duração de combustão $(\mathrm{MDC})(\mathrm{r}=-0,7)$ e altura média da chama (MHC) $(\mathrm{r}=-0,6)$.

Em estudo da inflamabilidade de combustíveis florestais marroquinos, Hachmi et al. (2011) apontaram elevada correlação entre o teor de umidade e os parâmetros relacionado à inflamabilidade do fogo, exceto para MDC. Por sua vez, Batista et al. (2012), ao avaliarem a inflamabilidade de espécies para uso em cortina de segurança no sul do Brasil, observaram correlação entre o teor de umidade e parâmetros de combustão, o que, segundo os autores, demonstra a importância da umidade da vegetação na ignição e propagação do fogo. Porém, Petriccione (2006), em avaliação da inflamabilidade de espécies do Mediterrâneo, afirmou não ter encontrado correlação entre o U\% e o MTI, assim como entre o U\% e a MDC.

O índice de combustão (IC) e o valor de inflamabilidade (VI) das espécies estudadas estão apresentados na Tabela 4.

As demais espécies estudadas indicaram nível " 0 " de inflamabilidade, sendo classificadas como fracamente inflamáveis. Destas, S. terebinthifolius e B. glabra apresentaram uma intensidade de combustão baixa $(\mathrm{IC}=2)$.

L. lucidum apresentou índice médio de intensidade de combustão ( $\mathrm{IC}=3$ ) e foi classificada como fracamente inflamável (VI $=0)$. Batista \& Biondi (2009), ao avaliarem a inflamabilidade desta espécie, a classificaram como não inflamável (material verde) e de baixa inflamabilidade (material seco em estufa), sendo observado pelos autores dificuldade de ignição e propagação do fogo, o que indica potencial para o uso em cortina de segurança.

Pesq. flor. bras., Colombo, v. 36, n. 88, p. 387-391, out./dez. 2016
Tabela 4. Índice de combustão (IC) e valor de inflamabilidade (VI).

\begin{tabular}{lcc}
\hline \multicolumn{1}{c}{ Espécie } & IC & VI \\
\hline Pinus taeda & 5 & 3 \\
Psidium cattleianum & 4 & 0 \\
Ligustrum lucidum & 3 & 0 \\
Schinus terebinthifolius & 2 & 0 \\
Bougainvillea glabra & 2 & 0 \\
\hline
\end{tabular}

P. taeda apresentou maior índice de combustão $(\mathrm{IC}=5)$, sendo classificado como muito alto, e maior valor de inflamabilidade (VI = 3), classificando a espécie como inflamável. Petriccione (2006) avaliou outras duas espécies de Pinus: P. halepensis, classificado como moderadamente inflamável, e $P$. pinaster, classificado como pouco inflamável.

Ganteaume et al. (2013), ao avaliarem a inflamabilidade de espécies ornamentais no sudoeste da França, apontaram as espécies Pittosporum tobira e Cupressus sempervirens como fracamente e pouco inflamável, respectivamente, e não recomendaram o uso de Phyllostachys sp., Prunus laurocerasus e Photinia fraseri (fotínia), por apresentarem alta inflamabilidade. Os autores relacionaram os resultados de inflamabilidade com a calorimetria e características físicas foliares das espécies, o que, segundo eles, auxiliou a compreensão dos resultados. Os pesquisadores ainda sugerem experimentos de queima utilizando plantas inteiras, para confirmar os resultados e torná-los amplamente aplicáveis, assim como uma padronização dos protocolos para determinação da inflamabilidade e o estabelecimento de uma classificação comum para os resultados.

\section{Conclusão}

As espécies Bougainvillea glabra e Schinus terebinthifolius apresentaram melhores resultados em relação às variáveis de inflamabilidade. Entretanto, as quatro espécies estudadas (Psidium cattleianum, Ligustrum lucidum, S. terebinthifolius e B. glabra) foram classificadas como fracamente inflamáveis, enquanto Pinus taeda demonstrou ser inflamável, indicando, em princípio, potencial das mesmas para uso em cortinas de segurança na prevenção de incêndios florestais. Porém, recomenda-se que essas sejam submetidas a análises de combustibilidade e de calorimetria em estudos futuros, a fim de corroborar com os resultados deste estudo.

\section{Referências}

Alessio, G. A. et al. Influence of water and terpenes on flammability in some dominant Mediterranean species. International Journal of Wildland Fire, v. 17, n. 2, p. 274-286, 2008. DOI: 10.1071/ WF07038. 
Batista, A. C. \& Biondi, D. Avaliação da inflamabilidade de Ligustrum licidum Aiton (Oleaceae) para uso potencial em cortinas de segurança na região sul do Brasil. Revista Brasileira de Ciências Agrárias, v. 4, n. 4, p. 435-439, out/dez, 2009. DOI: 10.5039/agraria.v4i4a11.

Batista, A. C. et al. Evaluación de la inflamabilidad de árboles y arbustos utilizados en la implementación de barreras verdes en el sur del Brasil. In: González-Cabán, A. (Coord.). Proceedings of the Fourth International Symposium on Fire Economics, Planning, and Policy: Climate Change and Wildfires. Albany, CA: U.S. Department of Agriculture, Forest Service, Pacific Southwest Research Station, 2012. p. 256-264. Disponível em: $<$ http://www. fs.fed.us/psw/publications/documents/psw_gtr245/>. Acesso em: 13 abr. 2015.

Batista, A. C. Incêndios florestais. Recife: Imprensa Universitária da UFRPE, 1990. 115 p.

Ganteaume, A. et al. Flammability of some ornamental species in wildland-urban interfaces in southeastern France: Laboratory Assessment at Particle Level. Environmental Management, v. 52, p. 467-480, 2013. DOI: 10.1007/s00267-013-0067-z.

Hachmi, M. et al. A Simple technique to estimate the flammability index of Moroccan forest fuels. Journal of Combustion, ID 263531 , 2011, 11 p. DOI: 10.1155/2011/263531.
Haltenhoff, H. Silvicultura preventiva. 2. ed. Santiago de Chile: Ministerio de Agricultura, Corporación Nacional Forestal, 2006. 40 p. (Manual técnico, n. 452).

Hernando, C. L. Combustibles forestales: inflamabilidad. In: Vélez, R. M. (Coord). La defensa contra incêndios forestales: fundamentos y experiências, 2. ed. Espanha: Mcgrawhill, 2009.

Petriccione, M. Infiammabilità della lettiera di diverse specie vegetali di ambiente Mediterraneo. 2006. 48 f. Tese (Doutorado em Biologia Aplicada) - Dipartimento di Biologia Strutturale e Funzionale, Università Degli Studi Di Napoli Federico II, Napoli.

Ribeiro, G. A. et al. Uso de vegetação como aceiro verde na redução da propagação de fogo sob linhas de transmissão: relatório técnico. 2007.

Soares, R. V. \& Batista, A. C. Incêndios florestais: controle, efeitos e uso do fogo. Curitiba, 2007. 264 p.

Soares, R. V. Novas tendências no controle de incêndios florestais. Revista Floresta, v. 30, n. 1/2, p. 11-21, 2000.

Valette, J. C. Inflammabilities of mediterranean species. Porto Carras: Research and Development of the European Commission European School of Climatology and Natural Hazards, 1992. 12 p. (Document PIF9208). 
